\title{
Efecto del tamaño de grano en la corrosión de la aleación $\mathrm{Al}-1 \% \mathrm{Mn}^{\left({ }^{\circ}\right)}$
}

Palabras clave: Aleación de aluminio. Tamaño de grano. Corrosión general. Corrosión por picaduras.

\section{Effect of the grain size on the corrosion behaviour of an Al-1\% Mn alloy}

\begin{abstract}
The influence of the grain size on the corrosion behaviour of an $\mathrm{Al}-1 \% \mathrm{Mn}$ alloy has been investigated in sodium chloride solution through the evaluation of corrosion, repassivation and pitting potentials respectively. Potentiodynamic polarization curves including scratched techniques were also employed. The attack morphology was analyzed by scanning electronic microscopy. Results show that corrosion parameters were not dependent on the alloy microstructure. Micrographies show that the pitting morphology is independent of the alloy grain size and that when the chloride concentration increases, localized attack also increases.
\end{abstract}

Keywords: Aluminium alloys. Grain size. General corrosion. Pitting corrosion.

\section{INTRODUCCIÓN}

Una serie de estudios realizados sobre el aluminio y sus aleaciones en el campo de la corrosión (15) han demostrado el excelente comportamiento de estos materiales en servicio, principalmente por la formación de productos de corrosión estables y protectores. No obstante, la ausencia de oxígeno a valores de $\mathrm{pH}$ bajos y altos o la presencia de iones cloruro da lugar a la rotura de la película formada.

Las aleaciones de Al-Mn son posiblemente las más importantes entre las no endurecidas por envejecimiento y generalmente contienen entre 0,25 y $1,25 \%$ de Mn. En estas aleaciones se encuentran presentes como impurezas hierro y silicio y como

(*) Trabajo recibido el día 26 de junio de 1996.

Instituto de Química, Instituto de Física. Universidad Católica de Valparaíso. Casilla 4059. Valparaíso (Chile). aleantes minoritarios cobre, magnesio, cromo y cinc. Este material se utiliza ampliamente en equipos químicos y en aplicaciones arquitectónicas por su alta resistencia a la corrosión.

Los tratamientos mecánicos y térmicos aplicados a la aleación determinan el tipo y la condición de la película protectora, de lo que se deduce que el grado del ataque depende de la composición, historia metalúrgica y condiciones de servicio del material.

Desde el punto de vista metalúrgico (6-8), los diversos aspectos microestructurales que caracterizan un material, tales como tamaño y orientación de los granos; tamaño, forma y distribución de las partículas de fases secundarias, etc., pueden dar lugar a una evolución distinta del potencial entre las diferentes áreas del metal y, consecuentemente, al desarrollo de zonas anódicas y catódicas. Investigaciones realizadas sobre el tema (9-12) indican una dependencia entre el tamaño de grano y el valor del potencial de corrosión, observándose un 
desplazamiento hacia valores positivos de potencial a medida que el tamaño de grano disminuye, pero no tiene influencia en el potencial de picadura.

El presente trabajo tiene como objetivo continuar dichas investigaciones y así poder proyectar la fabricación de aleaciones con un determinado tamaño de grano, que ofrezcan un mejor comportamiento mecánico y de resistencia a la corrosión frente a medios agresivos. Dicho estudio evalúa el potencial de corrosión, de repasivación y de picadura en diferentes concentraciones de cloruro de sodio $(\mathrm{NaCl})$, en función del tamaño de grano de la aleación. La morfología del ataque se determina por microscopía electrónica de barrido.

\section{PARTE EXPERIMENTAL}

El material empleado es la aleación comercial Al-1 \% Mn, cuya designación comercial es 3003; se obtuvo mediante colada continua y la composición química, determinada por absorción atómica, se muestra en la tabla I.

Esta aleación corresponde a materiales que se han endurecido, únicamente por deformación en frío, de tal forma que las propiedades mecánicas deseadas se alcanzan sin necesidad de tratamientos térmicos suplementarios que den lugar a la precipitación de fases secundarias.

La aleación se ha tratado termomecánicamente (12) con el fin de lograr diferentes tamaños de grano recristalizado, entre 147 y $320 \mu \mathrm{m}$, sin que ocurra un cambio significativo de la forma, tamaño y distribución de las partículas gruesas de fases secundarias.

Los ensayos se realizaron empleando soluciones $0,01 \mathrm{M}, 0,1 \mathrm{M}, 0,6 \mathrm{M}$ y $1,0 \mathrm{M}$ de $\mathrm{NaCl}$, desaireadas con nitrógeno (13) en una celda convencional por la cual circulaba agua a $25^{\circ} \mathrm{C}$ proveniente de un termostato. El potencial de corrosión se obtiene al registrar el valor del potencial a circuito abierto que adquiere el sistema durante $120 \mathrm{~min}$, tiempo suficiente para que el potencial de corrosión alcance un valor estable. El potencial de picadura se determinó utilizando la técnica de raspado (14), y el de repasivación mediante curvas de polarización en dirección anódica y catódica (15).

Para las medidas anteriores se empleó un potenciostato, un registrador y un generador con una

TABLA I.- Composición de la aleación Al-1 \% Mn, en $\%$ en masa

TABLE I.- Al $1 \%$ Mn alloy composition, \% in mass

\begin{tabular}{|c|c|c|c|c|c|c|}
\hline $\mathrm{Al}$ & $\mathrm{Mn}$ & $\mathrm{Fe}$ & $\mathrm{Si}$ & $\mathrm{Cu}$ & $\mathrm{Zn}$ & $\mathrm{Cr}$ \\
\hline Rest. & 0,925 & 0,645 & 0,23 & 0,14 & 0,04 & 0,001 \\
\hline
\end{tabular}

velocidad de barrido de 1 (mV/s). Como contraelectrodo se usó un alambre de platino de gran área $y$, como electrodo de referencia, uno de calomelanos saturado. Las probetas, de $1 \mathrm{~cm}^{2}$ de área expuesta, se pulieron con papel de $\mathrm{SiC} 600$, se lavaron con agua destilada y se secaron al aire. Además, se determinó la morfología del material atacado mediante microscopía electrónica de barrido con un equipo Philips 515.

\section{RESULTADOS Y DISCUSIÓN}

En la tabla II se indican los tamaños de grano de las muestras utilizadas en esta investigación y las deformaciones correspondientes (12).

En las figuras 1 y 2 se muestra la microestructura correspondiente a las probetas de menor y mayor tamaño de grano, respectivamente.

El sistema Al-1 \% Mn/solución agresiva a circuito abierto se comporta como un metal en el cual se forma y se estabiliza una película protectora; esto se puede deducir de la representación gráfica potencial de corrosión/tiempo, que se muestra en la figura 3, en la cual se observa que al introducir las probetas de diferentes tamaños de grano en la solución se tiene un potencial de corrosión inicial

TABla II.- Tamaño de grano en función de la deformación efectiva aplicada (12)

TABLE II.- Grain size versus the effective stress applied (12)

\begin{tabular}{|c|c|c|c|c|}
\hline$\epsilon$ & 2,33 & 0,20 & 0,15 & 0,11 \\
\hline $\mathrm{d}, \mu \mathrm{m}$ & $24 \times 2$ & $147 \times 2$ & $216 \times 17$ & $320 \times 25$ \\
\hline
\end{tabular}

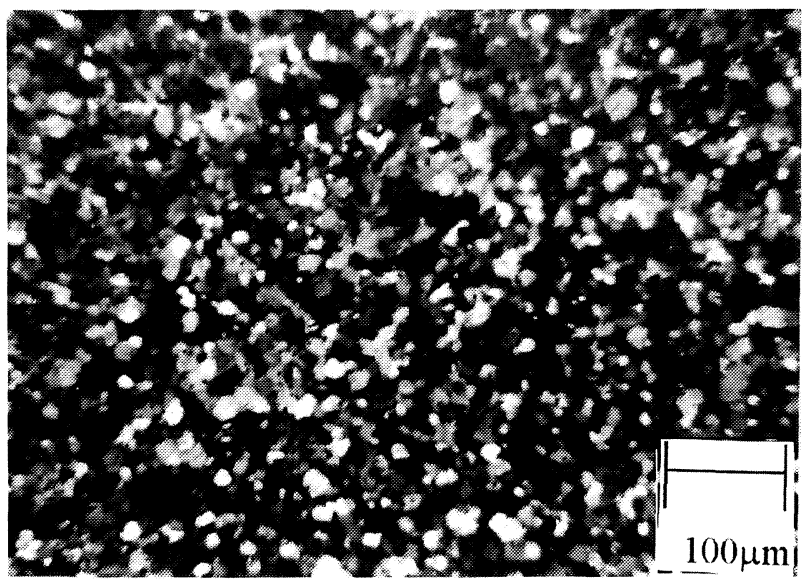

FIG. 1.- Micrografía de Al-1 \% Mn, $d=24 \mu \mathrm{m}$ (12).

FIG. 1.-Micrography of the Al-1 \% Mn alloy. d = $24 \mu m(12)$. 


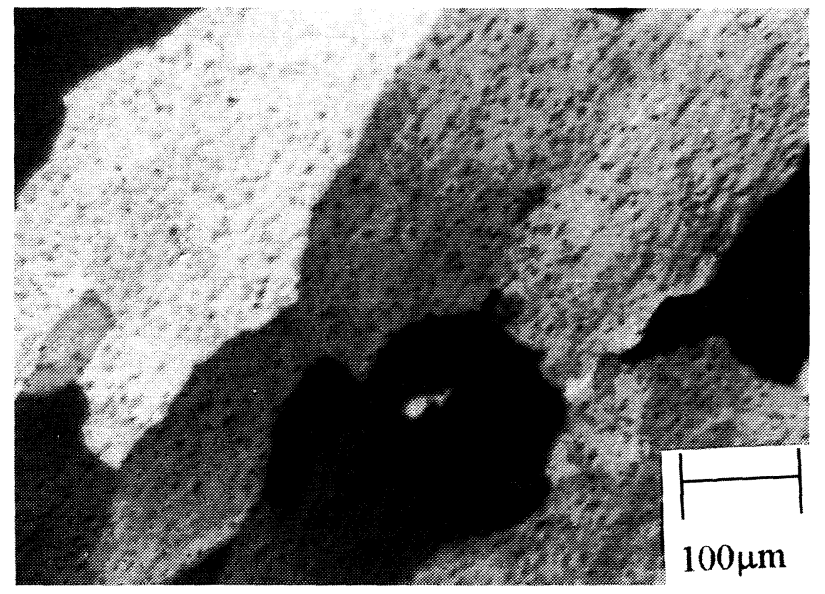

FIG. 2.- Micrografía de Al-1 \% Mn, $d=320 \mu \mathrm{m}$ (12).

FIG. 2.- Micrography of the Al-1\% Mn alloy. $\mathrm{d}=$ $320 \mu \mathrm{m}(12)$.

negativo, pero, transcurrido cierto tiempo, el potencial alcanza un valor estacionario superior al primero; este comportamiento es similar para las probetas con tamaños de grano diferentes. Es importante destacar que el estado superficial previo del electrodo influye en los valores del potencial, por lo que se debe cuidar su preparación para obtener resultados reproducibles. El valor de potencial promedio de corrosión de la aleación tratada termomecánicamente después de estar sumergida durante $2 \mathrm{~h}$ en $\mathrm{NaCl} 1 \mathrm{M}$, fue de $0,99 \mathrm{~V}_{\mathrm{ECS}}$, valor que concuerda con los obtenidos por otros autores (16 y 17).

En la figura 4 se compara el potencial de corrosión $\left(E_{c}\right)$, en función del tamaño de grano para las distintas concentraciones de anión agresivo. En

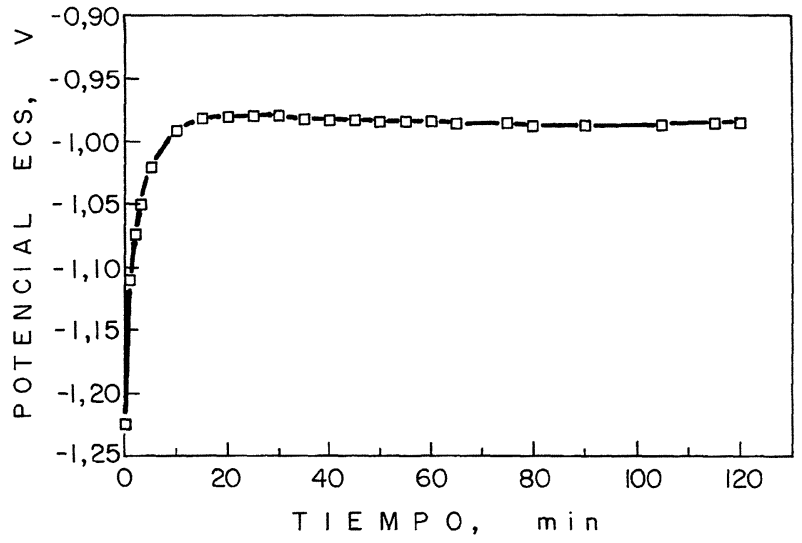

Fig. 3.- Potencial de corrosión $\left(E_{c}\right)$ en función del tiempo; $\mathrm{NaCl} 1 \mathrm{M}$.

FIG. 3.- Corrosion potential $\left(E_{c}\right)$ versus time in $\mathrm{NaCl} 1 \mathrm{M}$.

ellas se observa que el $E_{c}$ decrece ligeramente con el incremento de la concentración de cloruro, disminuyendo así la zona de inmunidad del material.

En la misma figura se muestra, además, la variación del potencial de picadura $\left(E_{p}\right)$ y de repasivación $\left(E_{r}\right)$. Se aprecia que tanto los valores de $E_{p}$ como de $E_{r}$ no parecen depender del tamaño de grano de la aleación, pero sí de la concentración de haluro. En general, se observa que, a medida que aumenta la concentración del anión, $E_{p}$ y $E_{r}$ alcanzan valores más negativos y la diferencia entre ambos se hace menor, indicando una disminución en la zona de pasividad de la aleación y haciendo que en estas condiciones la aleación sea más susceptible a la corrosión por picado. Además, esta diferencia es similar para muestras de distintos

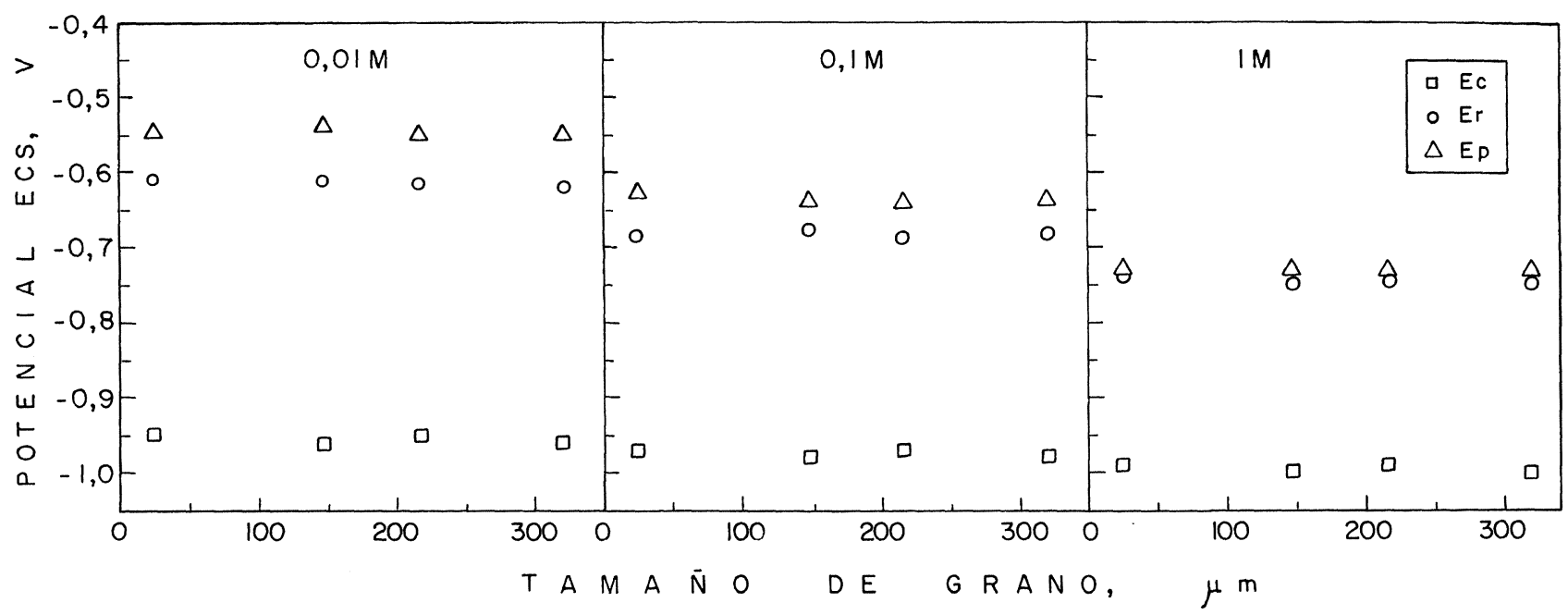

FIG. 4.- Potencial de corrosión $\left(E_{c}\right)$, potencial de picadura $\left(E_{p}\right)$ y potencial de repasivación $\left(E_{r}\right)$, en función del tamaño de grano para diferentes soluciones de $\mathrm{NaCl}$ con: a) $0,01 \mathrm{M}$, b) $0,1 \mathrm{M}$, y c) 1,0M.

FIG. 4.- Corrosion potential $\left(E_{c}\right)$, pitting potential $\left(E_{p}\right)$ and repasivation potential $\left(E_{r}\right)$ versus grain size for different ClNa concentrations: a) $0.01 \mathrm{M}$, b) $0.1 \mathrm{M}$, and c) $1.0 \mathrm{M}$. 


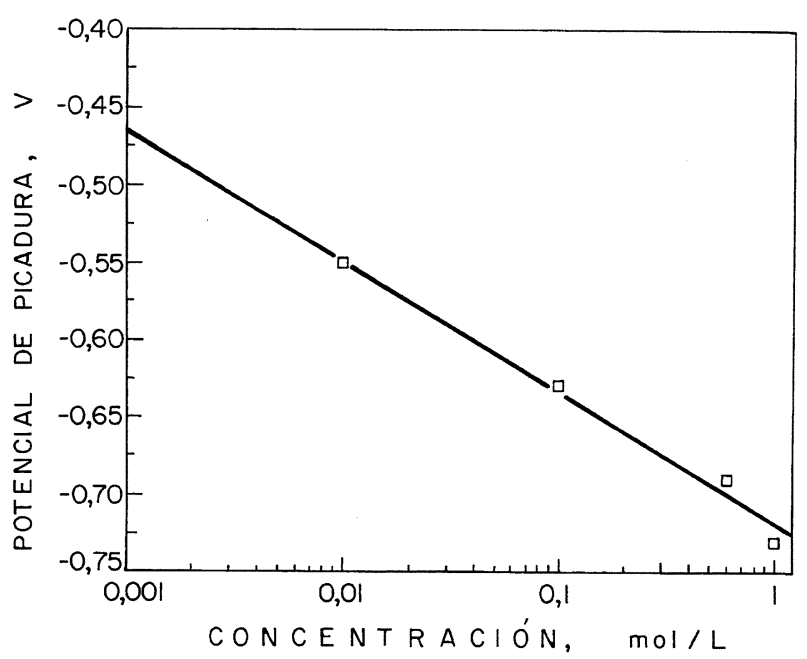

FIG. 5.- Potencial de picadura en función de la concentración de ion cloruro para distintos tamaños de grano.

FIG. 5.- Pitting potential versus ion chloride concentration for different grain sizes.

tamaños de grano, lo que indica que el ataque localizado sufrido por la aleación es independiente de la microestructura del material base. Por otra parte, la diferencia entre $E_{p}$ y $E_{r}$ se hace mínima a medida que aumenta la concentración del anión, lo cual hace disminuir la susceptibilidad del material a la corrosión en rendijas, desapareciendo la zona de pasividad imperfecta.

En esta investigación se fuerza, polarizando, la formación de picaduras, pero si se tiene en cuenta que, incluso para la mayor concentración de cloruros entre las ensayadas $(1 \mathrm{M})$, la diferencia entre $E_{c}$ y $E_{p}$ es de unos $250 \mathrm{mV}$ y que crece hasta unos 400 $\mathrm{mV}$ para la concentración menor (0,01M, Fig. 4),

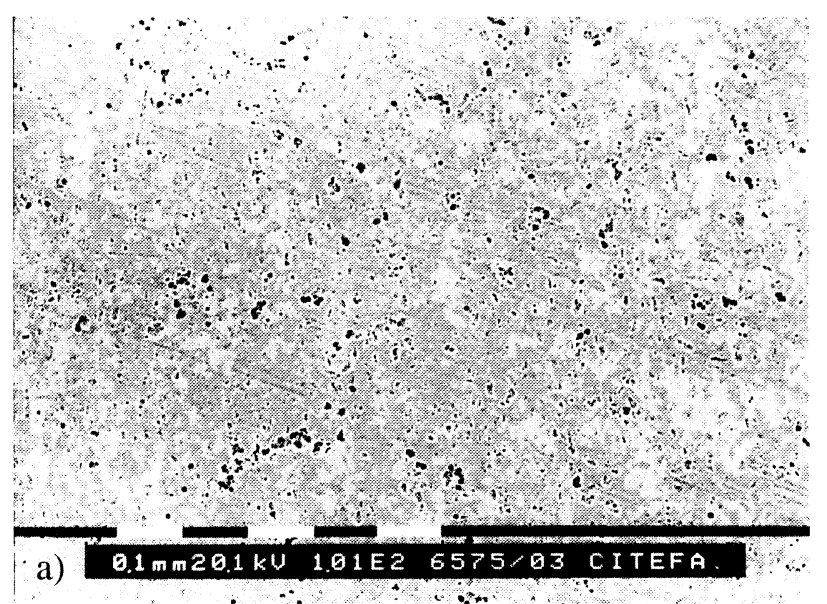

habría que contar con una apreciable resistencia a la corrosión por picaduras de la aleación estudiada en condiciones naturales de exposición (sin polarizar). Además, las diferencias entre $E_{p}$ y $E_{r}$ son siempre reducidas, de manera que, ante roturas eventuales de la película pasivante, es de esperar una fácil repasivación.

El potencial de picado, generalmente, es función de factores tales como composición de la aleación, historia metalúrgica del material y medio agresivo. En esta investigación no se observaron variaciones de $E_{p}$ con el tamaño de grano, pero sí existe una dependencia con el medio agresivo expuesto. De la representación de $E \mathrm{p}$ en función de la concentración de anión para los distintos tamaños de grano, mostrada en la figura 5 , se obtiene una curva que obedece a la siguiente ley:

$$
E_{p}=-0,719+0,086 \mathrm{pCl}^{-}
$$

Este tipo de relación entre el potencial de picadura y la concentración del anión se conoce tanto para el aluminio como para otros metales. Habitualmente, los valores de la pendiente son del orden de $0,07 \pm 0,01$ (V/década), sin embargo, para el aluminio y sus aleaciones se pueden obtener valores diferentes dependiendo tanto de las características del metal (composición) como del método empleado.

Al comparar los resultados de otros autores (1619) con los obtenidos en este trabajo, se tiene una coincidencia bastante aceptable, tanto del valor de $E_{p}$ para soluciones $1 \mathrm{M} \mathrm{de} \mathrm{Cl}^{-}$como de la pendiente de la recta obtenida. Esto indicaría que el tratamiento termomecánico a que se sometió a la aleación, y que generó diferentes microestructuras, ejerce una influencia mínima sobre el potencial de picadura del material, como se muestra en la figura 6 . En

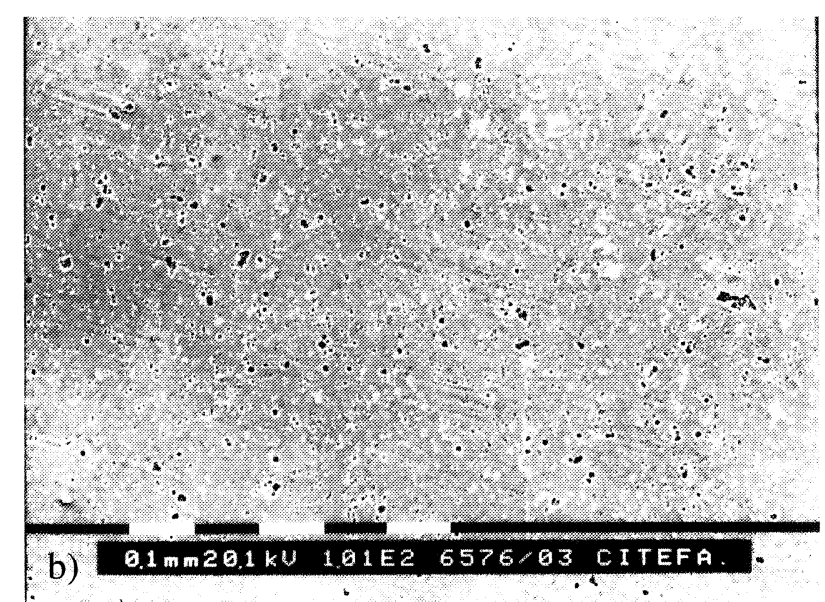

FIG. 6.- Micrografías de muestras de diferentes tamaños de grano atacadas en solución $1 \mathrm{M} \mathrm{NaCl}, \mathrm{pH}$ 1,5: a) $24 \mu \mathrm{m}$, b) $147 \mu \mathrm{m} . \times 100$.

FIG. 6.- Micrographs of samples of different grain sizes attacked in $1 \mathrm{M} \mathrm{NaCl}$ solution $(p H=1.5):$ a) $24 \mu \mathrm{m}$, b) $147 \mu \mathrm{m} . \times 100$. 

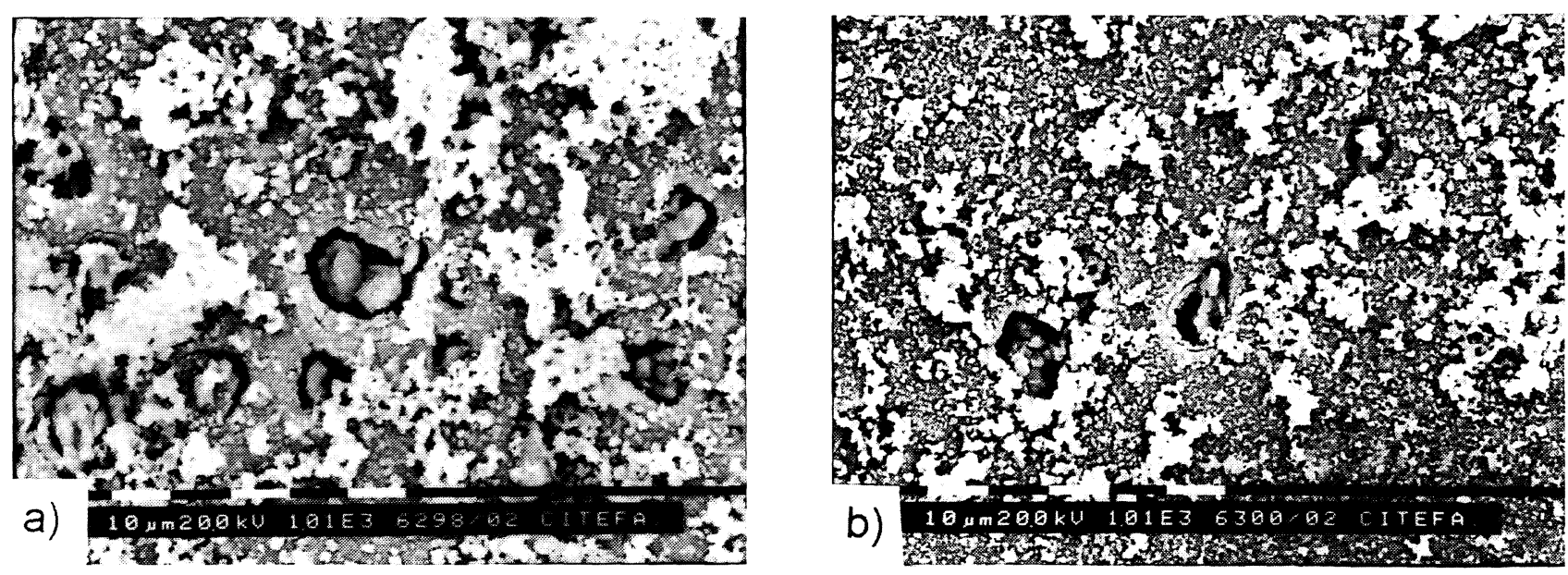

FIG. 7.- Micrografías de muestras de diferentes tamaños de grano atacadas en solución $0,01 \mathrm{M} \mathrm{NaCl}, \mathrm{pH}$ 1,5: a) $24 \mu \mathrm{m}$, b) $216 \mu \mathrm{m} . \times 1.000$.

FIG. 7.- Micrographs of samples of different grain sizes attacked in 0.01M NaCl solution $(p H=1.5):$ a) $24 \mu m$, b) $216 \mu \mathrm{m} . \times 1,000$.

del material, como se muestra en la figura 6. En ella, se observa que el ataque localizado se distribuye de forma similar en materiales con diferentes tamaños de grano $(25$ y $147 \mu \mathrm{m})$ y que el número de picaduras es prácticamente independiente de la microestructura del metal.

En la figura 7 se muestran aspectos de los productos de corrosión y el tipo de ataque en probetas con tamaños de grano de $24 \mu \mathrm{m}$ y $216 \mu \mathrm{m}$, en una solución $0,01 \mathrm{M}$ de $\mathrm{NaCl}$. Por difracción de rayos X se determinó que los productos de corrosión están constituidos principalmente por $\mathrm{Al}_{2} \mathrm{O}_{3}$. En las micrografías de esta figura se observa que el ataque se distribuye heterogéneamente sobre la superficie, pudiéndose notar zonas con abundantes productos de corrosión, picaduras profundas y áreas libres, independientes del tamaño de grano del material. Por lo tanto, se puede inferir que el tamaño de grano no afecta a la morfología de las picaduras y tampoco a la nucleación en lugares específicos. La morfología del ataque de esta aleación es similar a la presentada en aluminio, lo que ya ha sido citado por otros autores (20 y 21).

En la figura 8 se observa el aspecto para una muestra de $24 \mu \mathrm{m}$ en una solución $1 \mathrm{M}$ de $\mathrm{NaCl}$. Al comparar las micrografías 7a) y 8 se observa que un aumento del contenido de cloruro produce un ataque mayor localizado sobre la aleación, incrementando el número de picaduras. También se puede distinguir que una vez producida la ruptura de la película pasiva, el ataque se extiende rápidamente y cubre grandes zonas de la superficie metálica, observándose además una coalescencia de picaduras. Este comportamiento fue similar en las muestras con diferentes tamaños de grano.

\section{CONCLUSIONES}

Los distintos tamaños de grano del aluminio Al$1 \% \mathrm{Mn}$, cuyo comportamiento se ha estudiado frente a la corrosión de esta aleación, no han presentado cambios de los parámetros medidos, como lo indican sus valores de potencial de corrosión, de picadura y de repasivación, que se mantienen prácticamente constantes para las diferentes microestructuras. Asimismo, a partir de las micrografías se puede concluir que la morfología de las picaduras es independiente del tamaño de grano del material. En cambio, se observa que un aumento de la concentración del medio agresivo incrementa el ataque localizado.

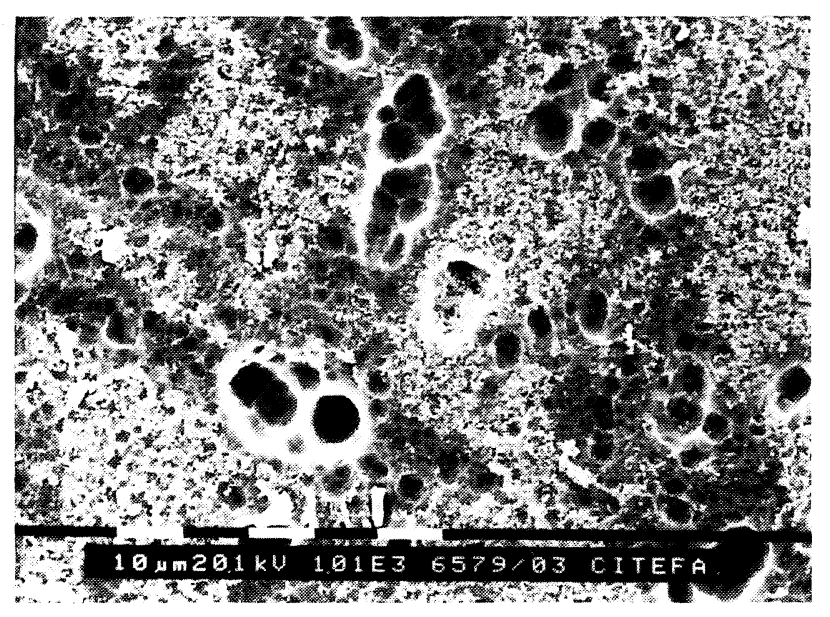

FIG. 8.- Micrografía de una muestra con tamaño de grano de $24 \mu \mathrm{m}$ atacada en solución $1 \mathrm{M} \mathrm{NaCl}$, $\mathrm{pH} 1,5 . \times 1.000$.

FIG. 8.-Micrograph of a sample with $24 \mu \mathrm{m}$ grain size attacked in a pH $1.51 \mathrm{M} \mathrm{NaCl}$ solution. $\times 1,000$. 


\section{Agradecimiento}

Los autores desean manifestar su agradecimiento a la Dirección General de Investigación de la Universidad Católica de Valparaíso por la financiación otorgada, y a la Dra. Blanca Rosales por su colaboración en la obtención de las micrografías con MEB en el CITEFA (Argentina).

\section{REFERENCIAS}

(1) Foley, R.T. Corrosion, 42 (5), 1986: 277-288.

(2) Bonewitz, R.A. Corrosion, 30 (2), 1974: 53-59.

(3) Godard, H.P. Corrosion, 20 (7), 1981: 9-15.

(4) Scully, J.C. "Treatise on Materials Science and Technology". Vol. 23 Corrosion. Aqueous processes and passive films. Ed. Academic Press. Nueva York, 1983: 1-53.

(5) Foroulis, Z.A. Proc. 5th International Congress of Metallic Corrosion. NACE. Tokio (Japón), 1972.

(6) Nes, E. Acta Metall., 24, 1976: 391-398.

(7) Zamin, M. Corrosion, 37 (11), 1981: 627-632.

(8) Lunder, O. y NisAncioglu, K. Corrosion, 44 (7), 1988: 414-422.

(9) VerA, R. Tesis M.Sc. en Metalurgia y Ciencia de los Materiales. Universidad Central de Venezuela. Caracas (Venezuela), 1990.
(10) Vera, R., Puchi, E.S. y Staia, M.H. Proc. Jornadas Metalúrgicas de la SAM y Segundo Congreso de ALAMET. Buenos Aires (Argentina), May., 1991.

(11) Vera, R., Staia, M.H. y Puchi, E.S. Proc. $4^{\circ}$ Congreso Iberoamericano de Corrosión y Protección. Mar del Plata (Argentina), 1992: 1-9.

(12) Staia, M., Puchi, E.S. y Vera, R. Acta Micros., 1 (1), 1992: 29-42.

(13) Gilroy, D. y Mayne, J. J. Appl. Chem., 12, 1962: 382.

(14) Pessall, N. y Liu, C. Electrochim. Acta, 16 (11), 1971: 1.987-2.003.

(15) Pourbaix, M. Atlas of Electrochemical Equilibria. Ed. Pergamon Press. Oxford, 1974.

(16) Beccaria, A.M. y Poggi, G. Br. Corros. J., 21 (1), 1986: 19-22.

(17) Sherif, K.P. y Narayan, R. Br. Corros. J., 24 (3), 1989: 199-203.

(18) RASPInI, Y.A. Corrosion, 49 (10), 1993: 821-828.

(19) Carroll, W.M. y Breslin, C.B. Br. Corros. J., 26 (4), 1991: 255-259.

(20) Elola, A.S., Otero, T.F. y Porro, A. Corrosion, 48 (10), 1992: 854-863.

(21) MCIntyre, J.F. y Dow, T.S. Corrosion, 48 (4), 1992: 309319. 